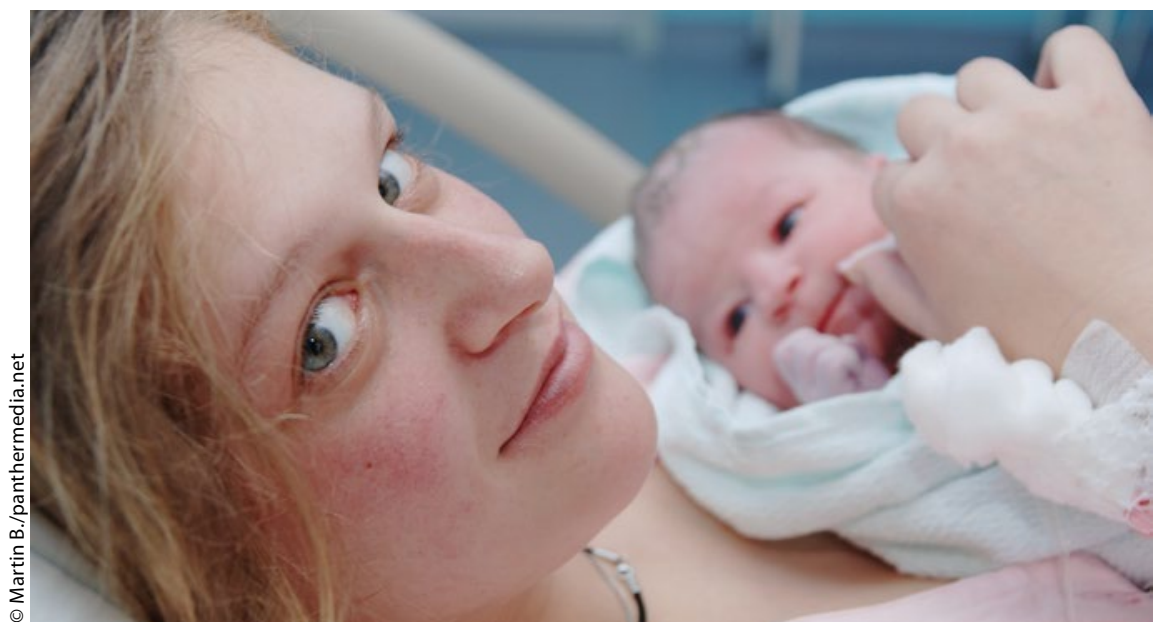

Geburten das Risiko für die Mutter, Signifikanz erreichte der Zusammenhang aber nicht. Auch ein Dammschnitt bot keinen statistisch relevanten Schutz. In der multivariaten Analyse blieben als Risikofaktoren für OASIS der Zangenein- satz, die Anwendung von vier oder mehr Handgriffen sowie das Woods' Manöver übrig. Interessanterweise war das Lösen des posterioren Kindesarms in der multivariaten Analyse kein signifikanter Gefahrenparamter. Falls das zuträfe, wäre es ratsam, nach Versagen des McRobertsManövers und des suprapubischen Drucks gleich das Lösen des hinteren Ärmchens zu versuchen, um den Damm der Mutter zu schützen. Die Autoren räumen allerdings ein, dass außer ihren Ergebnissen keine anderen Resultate für diesen Ratschlag sprechen und er somit womöglich nicht verallgemeinerbar ist. Die spezifische Kombination von Manövern, die als besonders riskant für die Entwicklung einer Sphinkterverletzung angesehen werden, in künftigen Studien herauszufinden, wäre klinisch bedeutsam, schreiben Gauthaman et al. Dann könnten in die Leitlinien zum Umgang mit Schulterdystokien auch Erwägungen besser einfließen, die perineale Verletzungen zum Inhalt hätten.

Dr. Robert Bublak

Gauthaman N et al. Shoulder dystocia and associated manoeuvres as risk factors for perineal trauma. Int Urogynecol J 2016;27:571-7

\section{Brustkrebs - Update der kardialen Sicherheitsanalyse zu Trastuzumab}

\section{Der monoklonale Antikörper Trastuzumab hat sich als tragende Säule in der adjuvanten Therapie des HER2-positiven Mammakarzinoms etabliert. Dem möglicherweise erhöhten Risiko für kardiotoxische Nebenwirkungen kommt daher große Bedeutung zu.}

W ie aus einer gemeinsamen Analyse der adjuvanten Studien NCCTG N9831 (Alliance) und NSABP B-31 (NRG) hervorgeht, verbesserte ein Jahr Trastuzumab-Therapie als Ergänzung zu Doxorubicin/Cyclophosphamid (AC) und nachfolgend Paclitaxel das krankheitsfreie und gesamte Überleben. Die Autoren legten bereits eine Analyse der N9831-Studie zur kardialen Sicherheit nach einem medianen Follow-up von 3,75 Jahren vor. Im Rahmen dieser Studie erhielten Patientinnen mit einem operablen, HER2-positiven Mammakarzinom vier AC-Zyklen, gefolgt von ausschließlich Paclitaxel (Arm A), Paclitaxel plus sequenziell Trastuzumab (Arm B) oder simultan Paclitaxel/Trastuzumab (Arm C). Die kumulative 3-Jahres-Inzidenz von kardialen Ereignissen (CE) betrug für die drei
Arme $0,3 \%, 2,8 \%$ und 3,3\%. Angesichts der überzeugenden Wirksamkeit von Trastuzumab führten die Autoren ein Update der kardialen Sicherheitsanalyse aus der N9831-Studie unter Einschluss von Patientinnendaten der B-31-Studie nach median 9,2 Jahren durch. Für die Evaluierung der kumulativen CE-Inzidenz und der linksventrikulären Ejektionsfraktion (LVEF) eigneten sich 1.944 Patientinnen, die eiwne Post-AC-Therapie durchliefen. Die mediane Nachbeobachtungszeit betrug 9,2 Jahre. 651 willigten einer Reevaluierung der LVEF nach einem sechsjährigen Follow-up ein. Ergebnis: absolute mediane Änderung der $\mathrm{EF}$ aus dem linken Ventrikel gegenüber dem Ausgangswert um -3,0\% im Arm A, $-2,5 \%$ im Arm B und $-3,0 \%$ im Arm C. Die kumulative CE-Inzidenz nach sechs
Jahren betrug in Arm A 0,6\% und 2,8\% bzw. 3,4\% in den Trastuzumab-haltigen Therapiearmen B und C. Nach median 9,2 Jahren Nachbeobachtung ergaben sich nur zwei zusätzliche Diagnosen von Herzinsuffizienz (CHF) im Vergleich zum 3,75-jährigen Follow-up. Die LVEF verbesserte sich bei der Mehrzahl der CHF-Patientinnen. Höheres Lebensalter über 60 Jahre, eine LVEF unter $65 \%$ und die Einnahme von Antihypertensiva korrelierten mit einem erhöhten Risiko für CE in den Studienarmen B und C.

Fazit: Die kumulative Inzidenz von kardialen Ereignissen erhöht sich geringfügig durch die Erweiterung einer adjuvanten Chemotherapie um Trastuzumab bei Frauen mit einem HER2-positiven Mammakarzinom. Spät auftrende Herzkomplikationen werden selten beobachtet. Der Nutzen von Trastuzumab im Kontext einer Anthrazyklin- und Taxan-basierten Chemotherapie überwiegt daher weiterhin das Risiko, so die Autoren.

\section{Wolfgang Zimmermann}

Advani PP et al. Long-Term Cardiac Safety Analysis of NCCTG N9831 (Alliance) Adjuvant Trastuzumab Trial. J Clin Oncol. 2016;34(6):581-7 